\title{
Erratum: Management of obstructive sleep apnea in an edentulous patient with a combination of mandibular advancement splint and tongue-retaining device: a clinical report
}

\author{
Huseyin Kurtulmus • H. Serdar Cotert
}

Received: 17 March 2008 / Accepted: 13 May 2008 /Published online: 13 January 2011

(C) Springer-Verlag 2011

Erratum to: Sleep Breath (2009) 13:97-102

DOI 10.1007/s11325-008-0201-y

This article has been retracted because of duplicate publication in different journals. 